\title{
Synchronous Detection of BPV and BVDV with Duplex Taqman qPCR Method
}

\author{
Zhuan-di Gong', Jin-jing Geng'2, Hao-qin Liang'2, Guo-zhen Lin'2, \\ Xiao-yun Shen ${ }^{3}$, Qion-yi Li ${ }^{4}$, Ruo-fei Feng ${ }^{4}$ \& Suo-cheng Wei ${ }^{3,4}$
}

\begin{abstract}
Background: Bovine parvovirus (BPV) and bovine viral diarrhea virus (BVDV) are commonly etiologies causing diarrhea in dairy herds. BPV is a member of Bocaparvovirus genus with a non-enveloped capsid. BVDV, belonging to Pestivirus genus in Flaviviridae, possesses a single-stranded RNA, and is classified into BVDV-1 and BVDV-2 genotypes according to the 5'UTR sequence. 21 genetic groups of BVDV-1 and four groups of BVDV-2 have been found. Diagnosis of viral diarrhea is often relied on virus detection by isolation or detection of serum antibody. The main objective of the present study was to establish a duplex real time PCR (qPCR) based on Taqman probe to detect synchronously BPV and BVDV. Materials, Methods \& Results: TaqMan probe and primers were designed and synthesized from the sequences of conserved $5^{\prime}$ - untranslated regions (5' UTR) of Haden strain of BPV and NADL strain of BVDV. The cDNAs were transcribed in vitro to make standard curves before optimizing the assay. DNA/PCR products were ligated into pMD18-T vector, and then used to transfer BL-21 competent cells to acquire the recombinant plasmids of pMD18-T-BPV and pMD18-T-BVDV. Optimum reaction conditions were comparatively selected. The sensitivity, specificity and reproducibility of TaqMan probe qRT-PCR were evaluated respectively. The results showed the concentrations of pMD18-T-BPV or pMD18-T-BVDV were $2.0 \times 10^{10} \mathrm{DNA}$ copies $/ \mu \mathrm{L}$, respectively. A duplex Taqman qPCR method was developed by optimizing the amplification conditions to simultaneously detect BPV and BVDV. The assay targets at highly conserved VP2 gene of BPV and 5' UTR gene of BVDV. This qPCR assay was assessed for specificity and sensitivity using DNA of BPV and cDNA of BVDV. For clinical validation, 308 samples were tested from clinically diarrhea calves. The results showed that optimum annealing temperature was achieved in 43.2 for duplex BPV and BVDV. Dynamic curves and standard curves were created following amplification of recombinant plasmids using the optimized duplex Taqman qPCR method, with an amplification efficiency of 95.69\%. Duplex Taqman qPCR could only detect DNA of BPV and cDNA of BVDV with a strong specificity. The detection limitation was as low as $2.0 \times 10^{1}$ copies $/ \mu \mathrm{L}$ of pMD18-T-BPV plasmid and $2.0 \times 10^{\circ} \mathrm{copies} / \mu \mathrm{L}$ for pMD18-TBVDV plasmid, respectively. Sensitivity of detection was 100 -fold higher than conventional PCR. Duplex Taqman qPCR had excellent repeatability or stability with less than $1.2 \%$ of intra-assay and inter-assay. 35 and 47 positive feces samples were identified using duplex Taqman qPCR in comparison to 30 and 42 positives for universal PCR, respectively.

Discussion: The bovine viral diarrhea virus (BVDV) is a key pathogenic factor in bovine diarrhea. Currently, few effective measures are available for the treatment or prevention for BVDV and BPV infections in animals. The technique was proven to be repeatable and linear over a range of at least 5 magnitudes, from $10^{1}$ to $10^{5} \mathrm{RNA} / \mathrm{DNA}$ copies, thus ensuring an accurate measurement of BPV DNA and BVDV RNA loads in clinical samples. In conclusion, a duplex Taqman qPCR was established for detecting simultaneously BPV and BVDV. Taqman qPCR method was rapid and specific assay. This assay was 100 -fold sensitive than conventional PCR. It will be propitious to rapidly and differentially diagnose pathogens of viral diarrhea of dairy farms.
\end{abstract}

Keywords: bovine parvovirus, bovine viral diarrhea virus, taqman probe, specificity, sensitivity.

DOI: $10.22456 / 1679-9216.93604$ 


\section{INTRODUCTION}

Bovine parvovirus (BPV) and bovine viral diarrhea virus (BVDV) are key pathogenic factors causing viral diarrhea of calves [2]. The genome of BPV comprises of single-stranded DNA $[6,23]$ and possesses three ORFs. ORF3 encodes VP1 and VP2 capsid viral proteins which share a C-terminal end [3]. VP2 region locates in the crystal structures $[4,15]$.

BVDV, belonging to Pestivirus genus in Flaviviridae, possesses a single-stranded RNA, and is classified into BVDV-1 and BVDV-2 genotypes according to the $5^{\prime}$ UTR sequence $[10,12]$. Up to date, at least 21 genetic groups of BVDV-1 and four groups of BVDV-2 have been found $[1,22]$. Understanding the variability of viral strains existing in a population provides valuable information of BVDV [10]. BVDV may cause various diarrhea symptoms in cattle, especially calves, which lead to the mortality increase of infected animals [9].

Several methods have been utilized to detect BPV and BVDV antigens [16,29]. However, each method has its weaknesses in terms of specificity and sensitivity $[14,17]$. Commonly used RT-PCR protocols require more than $1 \mathrm{~h}$ and a specialized PCR instrument [5,24]. Real time PCR (qPCR) offers superiority in comparison to conventional PCR method, also provide rapid and sensitive due to real time quantification of data and visualization [28]. Scarce specific detection is reported [18]. It is highly necessary to develop a more specific and precise protocol that may decrease operation time to less than $30 \mathrm{~min}$. In this study, we expect to develop a novel duplex qPCR based on the Taqman probe (Taqman qPCR), additionally to provide accurate and rapid method for detecting synchronously BPV and BVDV in dairy farms.

\section{MATERIALS AND METHODS}

Viruses

The viral strains used throughout this study included Haden strain of BPV and NADL strain of BVDV which were purchased from the America type culture collection (ATCC). Viruses were propagated at 37 and $5 \% \mathrm{CO}_{2}$ in MDCK cells using DMEM medium $^{1}$ and $8 \%$ newborn bovine serum ${ }^{2}$ for $5-7$ days. The supernatant was separated from medium fluids by centrifuging $2000 \times g$ for $10 \mathrm{~min}$. Viral DNA was extracted from the supernatant using the QIAamp MinElute virus spin $\mathrm{kit}^{3}$ referring to the manufacturer's instruction. DNA was quantified using SYBR Green, and then stored at -80 for the subsequent tests.

\section{Primers and probe design for $B P V$ and $B V D V$}

The full-length genomes of Haden strains of BPV (Accession: JN191349) and NADL strain of BVDV (Accession: AM709624.1, NC001461) were obtained from the GenBank database, respectively. One pair of universal primers (BPV-F/BPV-R, BVDV-F/ BVDV-R), one pair of specific primers (BPV-qF/qR) and one specific Taqman probe (BPV-probe, BVDVprobe) from $5^{\prime}$-untranslated region (5' UTR) genes of BPV and BVDV were designed, respectively using Primer 5.0 software. Both 5' end and $3^{\prime}$ end of the BPV-probe was labeled with CY5 and BHQ-2, respectively. The $5^{\prime}$ end and $3^{\prime}$ end of the BVDV-probe was also labeled with FAM and BHQ-1, respectively. The specificity of the designed primers and probes (Table 1) was verified with Primer-BLAST at NCBI online.

Table 1. Primers for BPV and BVDV amplification.

\begin{tabular}{|c|c|c|c|}
\hline Virus & Primer & Primer sequences $\left(5^{\prime} \rightarrow 3^{\prime}\right)$ & $\mathrm{bp}$ \\
\hline \multirow{5}{*}{ BPV } & BPV-F & GCGAAAACACGACTTTGGCT & \multirow{2}{*}{554} \\
\hline & BPV-R & GAGCCGTGTCACCAGTGTTA & \\
\hline & BPV-qF & CAAGCACATCCAATCAAC & \multirow{2}{*}{198} \\
\hline & BPV-qR & CCACAATGTTCTCGCTAA & \\
\hline & BPV-probe & CY5-CGTCCATCCGCCAGTTGAGTA-BHQ-2 & 198 \\
\hline \multirow{5}{*}{ BVDV } & BVDV-F & TCCTCYTYMGCGAMGGCC & \multirow{2}{*}{681} \\
\hline & BVDV-R & CTRCCAGTYAYTCTCCCDATYC & \\
\hline & $\mathrm{BVDV}-\mathrm{qF}$ & CCACTGTATCGCTACTAA & \multirow{2}{*}{197} \\
\hline & BVDV-qR & ССССТCTTTTATGTGGTA & \\
\hline & BVDV-probe & FAM-AGAGGAACATCTACAACCAC-BHQ1 & 681 \\
\hline
\end{tabular}


Construction of standard plasmids and copies

Viral genomic DNA was extracted from the supernatant using QIAamp MinElute virus spin $\mathrm{kit}^{3}$ referring to the manufacturer's instruction. The DNA was amplified by PCR using universal primer. Then, DNA was quantified using SYBR Green, and stored at -80 for the subsequent tests. The extracted RNAs of BVDV were reversely transcribed into cDNAs. The cDNAs were amplified by PCR using universal primers at 94 for $5 \mathrm{~min}$ and 35 cycles of 94 for $30 \mathrm{~s}$, 60 for $45 \mathrm{~s}, 72$ for $1 \mathrm{~min}$. DNA/PCR products were ligated into pMD18-T vector ${ }^{4}$ for $2 \mathrm{~h}$ at 16 , respectively. Then, BL-21 competent cells were transferred with the recombinant plasmids of DNA/PCR products and pMD18-T at 37 overnight. The recombinant plasmids were extracted using the EasyPureR Plasmid MiniPrep $\mathrm{Kit}^{5}$ and sequenced ${ }^{6}$. The sequences homology of the recombinant plasmids was compared with those of NCBI Genbank after they were sequenced by Shanghai Sangon Biotec, Shanghai, China. The recombinant plasmids of pMD18-T-BPV and pMD18-T-BVDV were acquired, respectively. Concentrations of pMD18-
T-BPV or pMD18-T-BVDV were $2.0 \times 10^{10}$ DNA copies $/ \mu \mathrm{L}$, respectively.

The plasmids concentrations were determined utilizing Ultramicro nucleic acid protein analyzer? DNA copies per $\mu \mathrm{L}$ were calculated based on the plasmid concentrations.

Optimization of $q P C R$ conditions and the establishment of standard curve

Optimization of the Taqman qPCR included prior assessment of the mono-specific PCR, testing several master mix reagent sets, titration of primer and probe concentrations and alteration of PCR cycling parameters. Initial assessment results showed that the optimum conditions of Taqman qPCR for BPV were $0.8 \mu \mathrm{L}$ probes $(18.0 \mu \mathrm{mol} / \mathrm{L}), 0.4 \mu \mathrm{L}$ primers $(10.0 \mu \mathrm{mol} / \mathrm{L})$ and annealing temperature of 43.2 for $40 \mathrm{~s}$. These for BVDV were $17.5 \mu \mathrm{mol} / \mathrm{L}$ of primer, $7.5 \mu \mathrm{mol} / \mathrm{L}$ of probe and annealing temperature of 51.4 for $40 \mathrm{sec}$, respectively. Based on the above conditions of single Taqman qPCR, the reaction systems of duplex Taqman qPCR for both BPV and BVDV were under 40 cycles of 95 for $30 \mathrm{~s}$, 95 for 5 s 43.2 - 47.0 for 30 s (Table 2).

Table 2. BPV and BVDV duplex qPCR reaction system.

\begin{tabular}{cc}
\hline Premiers and probes & Volume \\
\hline Taq Premix Ex Taq ${ }^{\mathrm{TM}}($ Probe qPCR $)(2 \times)$ & $10 \mu \mathrm{L}$ \\
BPV-qF $(14.5 \mu \mathrm{mol} / \mathrm{L})+$ BVDV-qF $(19.5 \mu \mathrm{mol} / \mathrm{L})$ & $0.4 \mu \mathrm{L}$ \\
BPV-qR $(14.5 \mu \mathrm{mol} / \mathrm{L})+$ BVDV-qR $(19.5 \mu \mathrm{mol} / \mathrm{L})$ & $0.4 \mu \mathrm{L}$ \\
BPV-qF $(18.0 \mu \mathrm{mol} / \mathrm{L})+$ BVDV-qF $(17.5 \mu \mathrm{mol} / \mathrm{L})$ & $0.8 \mu \mathrm{L}$ \\
BPV-qR $(18.0 \mu \mathrm{mol} / \mathrm{L})+$ BVDV-qR $(17.5 \mu \mathrm{mol} / \mathrm{L})$ & $0.8 \mu \mathrm{L}$ \\
ROX Reference Dye $(50 \times)$ & $0.2 \mu \mathrm{L}$ \\
DEPC H ${ }_{2} \mathrm{O}$ & $6.2 \mu \mathrm{L}$ \\
DNA $\left(2.0 \times 10^{6}\right.$ copies $\left./ \mu \mathrm{L}\right)$ & $2.0 \mu \mathrm{L}$ \\
\hline Total & $20.0 \mu \mathrm{L}$ \\
\hline
\end{tabular}

Mixed plasmids of pMD18-T-BPV and pMD18-T-BVDV were diluted by ten-fold from 2.0 $\times 10^{8}$ copies $/ \mu \mathrm{L}$ to $2.0 \times 10^{3}$ copies $/ \mu \mathrm{L}$. They were amplified with real-time PCR based on the optimized conditions for single PCR of BPV and BVDV. The annealing temperature of duplex Taqman qPCR was selected from 43.2 - 47.2 so as to establish the dynamics curve and standard curve, respectively.

\section{Specificity of Taqman $q P C R$}

The specificity of the Taqman qPCR was assessed by testing viral DNA of BPV and cDNA of BVDV. The cDNAs of other viruses were used as control, including Japanese encephalitis virus (JEV), Classical Swine Fever Virus (CSFV), Rabies virus (RABV), Bovine Rotavirus (BRV), Bovine Viral Diarrhea Virus (BVDV) and bovine foot and mouth disease virus (FMDV). All these viruses were provided or gifted by the State key laboratory of biological engineering and technology of the Northwest Minzu University and Lanzhou veterinary research institute of the Chinese Academy of Agricultural Sciences (Lanzhou, China). They are cultured in the common cells suitable to them, respectively. 
Sensitivity verifications of Taqman qPCR

Sensitivity of duplex Taqman qPCR was assessed using 10-fold dilutions of the mixed plasmid $\left(2.0 \times 10^{8}\right.$ to $2.0 \times 10^{0}$ copies $\left./ \mu \mathrm{L}\right)$ of $5^{\prime}$ UTR genes of BPV and BVDV. The standard curves were utilized to set for respective absolute quantifications . The results were compared with those of universal PCR so as to determine the minimum detection limit. The tests were performed in triplicate.

\section{Repeatability tests}

In order to verify the repeatability of duplex Taqman qPCR assay, the intra-assay and inter-assay were evaluated by testing the same plasmids at least three times in one experiment.

\section{Clinical applications}

We have collected 308 feces samples from diarrhea calves of six different cattle farms in Gansu Province of China. They were detected with Taqman probe qPCR and common PCR respectively, to verify the repeatability of this qPCR. Total RNA was extracted from feces using the same methods described above.

\section{RESULTS}

Optimization of annealing temperature for duplex Taqman BPV and BVDV

The results showed the least $\mathrm{Ct}$ and largest relative fluorescence units (RFU) were found in 43.2 fro duplex BPV and BVDV. Therefore, we could get 43.2 was the optimized annealing temperature.

\section{Dynamic and standard curves of duplex Taqman BPV and $B V D V$}

Both dynamic curves and standard curves were acquired after the different concentrations of recombinant plasmids $\left(2.0 \times 10^{8}\right.$ copies $/ \mu \mathrm{L}-2.0 \times 10^{3}$ copies $\left./ \mu \mathrm{L}\right)$ were amplified using the optimized duplex Taqman probe qPCR (Figure 1). The results showed a good linearity for pMD18-T-BPV $\left(\mathrm{R}^{2}=0.9987\right)$ and $\mathrm{pMD} 18-\mathrm{T}-\mathrm{BVDV}\left(\mathrm{R}^{2}\right.$ $=0.9998)$. The regression equations were as followings: $\mathrm{Y}_{(\mathrm{BPV})}=-3.477 \mathrm{gX}+40.023, \mathrm{Y}_{(\mathrm{BVDV})}=-3.536 \lg \mathrm{X}+44.429$, respectively. Amplification efficiency was $95.69 \%$.

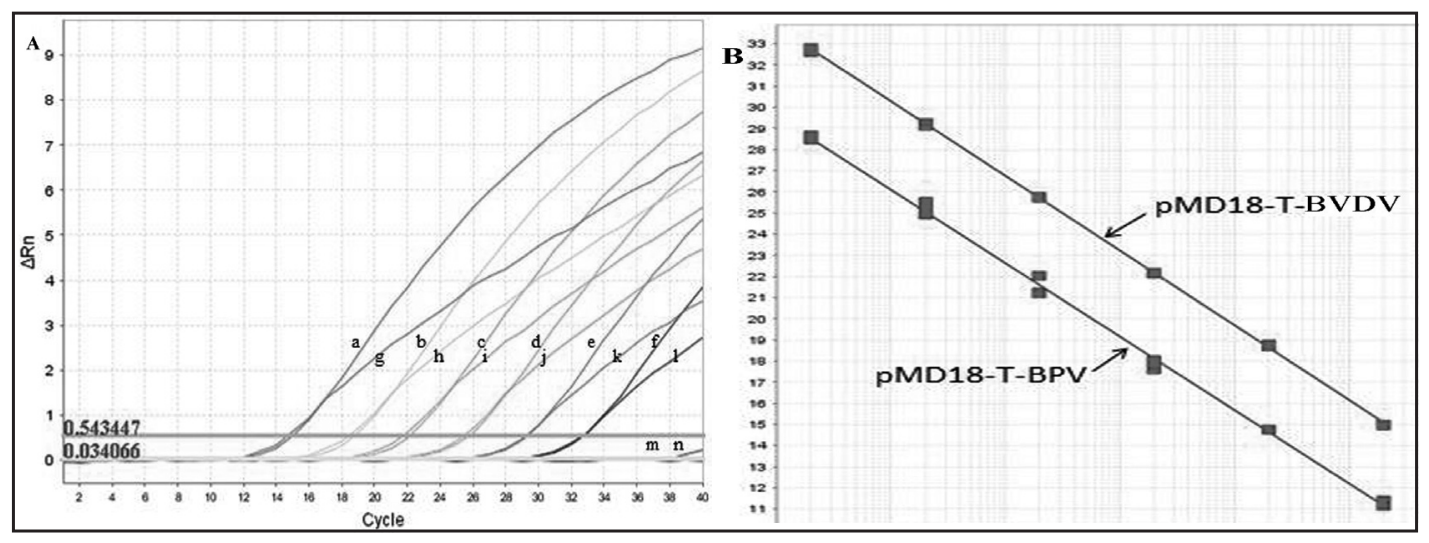

Figure 1. Dynamics curves (A) and standard curves (B) of BPV and BVDV duplex qPCR. a-f: $2.0 \times 10^{8}$ to $2.0 \times 10^{3}$ copies $/ \mu \mathrm{L}$ of pMD18-T-BVDV, respectively; g-1: $2.0 \times 10^{8}$ to $2.0 \times 10^{3}$ copies/ $\mu \mathrm{L}$ of pMD18-T-BPV, respectively; m-n: Negative control.

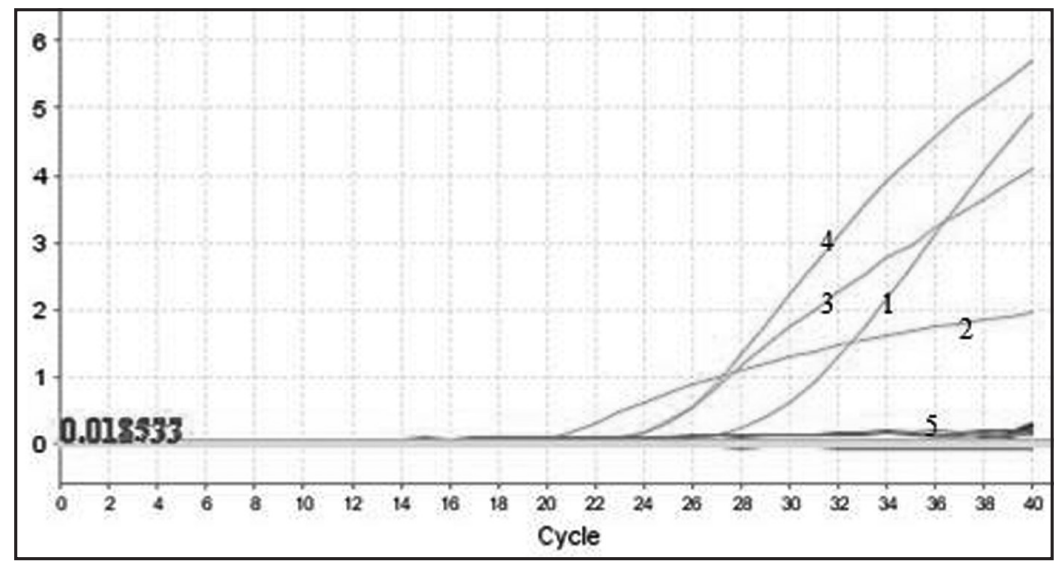

Figure 2. Specificity results of BPV and BVDV duplex qPCR. 1: BVDV; 2: BPV; 3-4, mixture of BPV and BVDV; 5: Control group (JEV, CSFV, RABV, BRV, FMDV and Negative control). 
Specificity assessments

As shown in Figure 2, duplex Taqman probe qPCR could clearly detect both DNA of BPV and cDNA of BVDV. The cDNA of other viruses displayed no signal bands, including JEV, CSFV, RABV, BRV and FMDV. The outcomes demonstrated that the established duplex probe qPCR had a strong specificity for BPV and BVDV.
Analytical sensitivity

Sensitivity assessment of the mixed plasmids showed that the least detection was $2.0 \times 10^{1}$ copies/ $\mu \mathrm{L}$ for $\mathrm{pMD} 18-\mathrm{T}-\mathrm{BPV}$ plasmid and $2.0 \times 10^{\circ}$ copies/ $\mu \mathrm{L}$ for pMD18-T-BVDV plasmid, respectively (Figure 3). Analytical sensitivity of Taqman qPCR was increased by 100 -fold in comparison with conventional PCR.

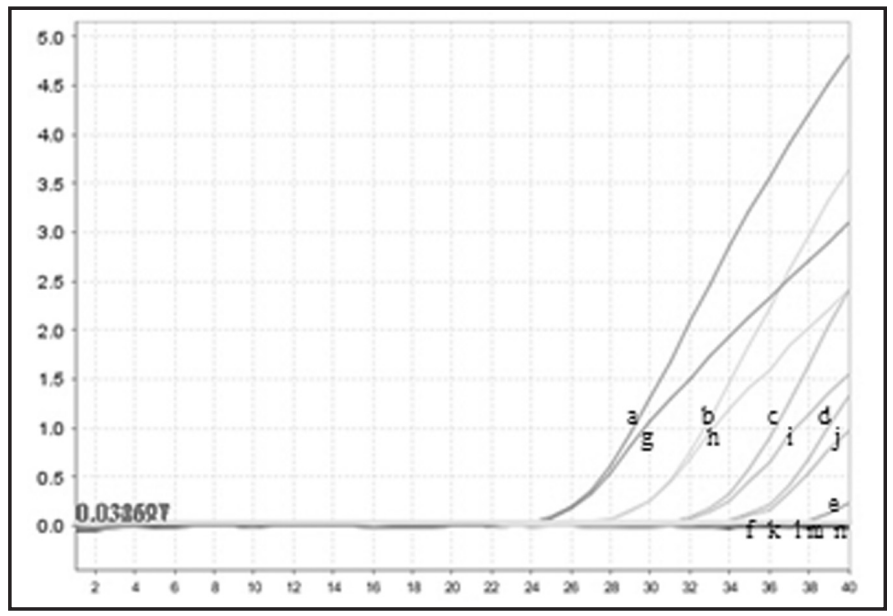

Figure 3. Sensitivity assay of BPV and BVDV duplex qPCR. a-f: $2.0 \times 10^{5}$ to $2.0 \times 10^{\circ}$ copies $/ \mu \mathrm{L}$ of pMD18-T-BVDV, respectively; g-l: $2.0 \times 10^{5}$ to $2.0 \times 10^{0}$ copies/ $\mu \mathrm{L}$ of pMD18-T-BPV, respectively; m-n: Negative control.

Repeatability of duplex Taqman BPVand BVDV

The results showed that Intra-assay and Interassay CVs were lower than $1.2 \%$, which indicated the developed duplex Taqman qPCR for BPVand BVDV had excellent repeatability or stability.

Table 3. Detection results of feces samples.

\begin{tabular}{cccc}
\hline PCR & $\begin{array}{c}\text { Feces } \\
\text { samples }\end{array}$ & $\begin{array}{c}\text { BPV } \\
\text { Positives }\end{array}$ & $\begin{array}{c}\text { BVDV } \\
\text { positives }\end{array}$ \\
\hline Duplex Taqman & 308 & 35 & 47 \\
Single (BPV) & 308 & 36 & \\
Single (BVDV) & 312 & & 49 \\
Universal & 308 & 30 & 43 \\
\hline
\end{tabular}

Evaluation of diagnostic performance

Three hundred and eight feces samples were detected using established duplex Taqman qPCR for the presence of BPV and BVDV of the diarrhea animals so as to find the specific pathogens. Results of Taqman qPCR were compared with those obtained by single PCR of BPV (or BVDV) and conventional PCR (Table 3). Specificity and accuracy of duplex Taqman qPCR were preferable to the single PCR and universal PCR, respectively.

\section{DISCUSSION}

The infection rate of bovine parvovirus (BPV) is about $83 \%$ to $100 \%$ worldwide $[6,19]$. The bovine viral diarrhea virus (BVDV) is a key pathogenic factor in bovine diarrhea [7,11]. Differential diagnosis of viral diarrhea is often relied on virus detection by isolation and/or detection of serum antibody using ELISA. Currently, few effective measures are available for the treatment or prevention for BVDV and BPV infections in animals [4,20]. Real-time PCR is now being used to identify and quantify many viral pathogens. RT-PCR methods for genotyping BVDV have been described previously $[26,28]$. However, this differs in the efficiency of RNA extraction, reverse transcription or PCR reactions, leading to unstable the sensitivity and specificity of this technique $[18,25]$. In order to overcome these faults, we designed one pair specificprimers (BPV-qF/qR and BVDV-qF/qR) and a specific Taqman probe (BPVprobe and BVDV-probe) targeting the highly conserved $5^{\prime}$-untranslated region (5' UTR) [20,21].

The specificity of Taqman qPCR was confirmed by the negative control and other viruses, 
including JEV, RABV, CSFV, BRV, BPIV, BAV and FMDV. The results showed that Taqman qPCR could only detect BPV and BVDV [21,27]. An internally controlled Taqman probe qPCR was developed and evaluated for detection and quantification of BPV and BVDV 5' UTR genes.

The technique was proven to be repeatable and linear over a range of at least 5 magnitudes, from $10^{1}$ to $10^{5}$ RNA/DNA copies, thus ensuring an accurate measurement of BPV DNA and BVDV RNA loads in clinical samples. The detection results of 308 feces samples demonstrated the Taqman duplex qPCR established in this study has more simple and fast, higher sensitivity and specificity than the universal PCR. These results are consistent with a previous study [12].

In addition, the 96-well format of the PCR plates ensures a high throughput, which detects simultaneously more than eight four samples. It will be useful for large-scale epidemiological surveys and be implemented to differentially diagnose BPV and BVDV infections in dairy farms.

\section{CONCLUSIONS}

A specific duplex Taqman qPCR was established for detecting BPV and BVDV in the present study. The optimum annealing temperature for duplex Taqman qPCR was 43.2. Minimum detection level of Taqman qPCR was 2.0 copies/ $\mu \mathrm{L}$ for BVDV and 20.0 copies/ $\mu \mathrm{L}$ for BPV. Its sensitivity was 100 -fold over the conventional PCR. The novel duplex Taqman
qPCR had excellent sensitivity, specificity and stability. It is will be conducive to enhancing diagnosis and therapy efficacy of diarrhea calves, and controlling effectively the spread of BPV and BVDV infections in cattle farms.

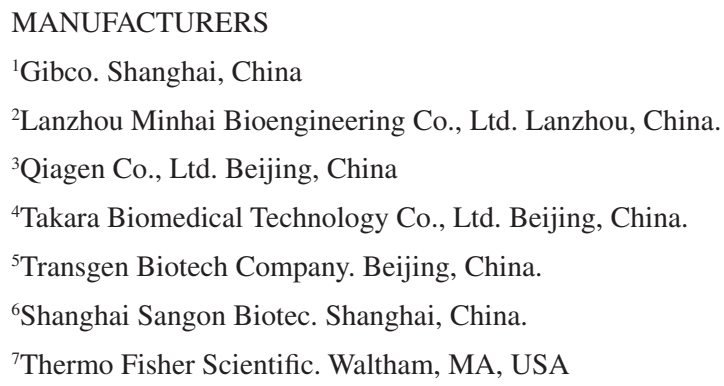

Funding. The work was supported by the Project of National Key research and Development Program of China in 13th Fiveyear Plan (2016-YFC0502601), the Science and technology support program project of Gansu province of China (Grant No: 1604NKCA044), the science and technology planning project of Lanzhou, China (Grant No:2016-3-120), Chengguan district science and technology planning project of Lanzhou, China (Grant: 2016-1-1), the innovative team development project of Ministry of education of China (IRT-17R88); the postgraduate student research innovation project of Northwest Minzu University (Yxm2016140).

Ethical approval. According to the Guidelines for the Care and Use of Animals of Northwest Minzu University, this study was carried out, and animal experiments were approved by Care and Use of Animals Center, Northwest Minzu University.

Declaration of interest. All authors have no conflicts of interest. The authors alone are responsible for the content and writing of the paper.

\section{REFERENCES}

1 Alpay G. \& Weșilag K. 2015. Serological relationships among subgroups in bovine viral diarrhea virus genotype 1 (BVDV- 1). Veterinary Microbiology. 175: 1-6.

2 Bauermann F.V., Flores E.F. \& Ridpath F.J. 2012. Antigenic relationships between Bovine viral diarrhea virus 1 and 2 and HoBi virus: possible impacts on diagnosis and control. Journal of Veterinary Diagnostic Investigation. 24: 253-260.

3 Chang J., Zhang Y., Yang D., Jiang Z., Wang F. \& Yu L. 2019. Potent neutralization activity against type O footand-mouth disease virus elicited by a conserved type $\mathrm{O}$ neutralizing epitope displayed on bovine parvovirus virus-like particles. Journal of General Virology. 100: 187-198.

4 Cibulski S.P., Teixeira T.F., Dos Santos H.F., de Sales Lima F.E., Scheffer C.M. \& Varela A.P. 2016. Ungulate copiparvovirus 1 (bovine parvovirus 2): characterization of a new genotype and associated viremia in different bovine age groups. Virus Genes. 52(2): 134-137.

5 de Los Angeles Ribas M., Tejero Y Cordero Y., Perez D Sausy A . \& Muller C.P. 2019. Identification of human parvovirus B19 among measles and rubella suspected patients from Cuba. Journal of Medical Virology. DOI: 10.1002/ jmv.25444.

6 de Souza W.M., Dennis T., Fumagalli M.J., Araujo J., Sabino-Santos G., Fgm M., Acrani G.O.. Aot C., Romeiro M.F. \& Modha S. 2018. Novel Parvoviruses from Wild and Domestic Animals in Brazil Provide New Insights into Parvovirus Distribution and Diversity. Viruses. 10(2): 143-148. 
7 Decaro N., Lanave G., Lucente M.S., Mari V., Varello K. \& Losurdo M. 2014. Mucosal disease-like syndrome in a calf persistently infected by Hobi-like pestivirus. Journal of Clinical Microbiology. 50(12): 2946-2954.

8 Decaro N., Martella V., Ricci D., Elia G., Desario C. \& Campolo M. 2005. Genotypespecific fluorogenic RT PCR assays for the detection and quantitation of canine coronavirus type I and type II RNA in faecal samples of dogs. Journal of Virological Methods. 130(1): 72-78.

9 Dubey P., Mishra N., Rajukumar K., Behera S.P., Kalaiyarasu S. \& Nema R.K. 2015. Development of a RT-PCR ELISA for simultaneous detection of BVDV-1 BVDV-2 and BDV in ruminants and its evaluation on clinical samples. Journal of Virological Methods. 213(1): 50-56.

10 Francisco J.D. 2017. Bovine viral diarrhea virus (BVDV) genetic diversity in Spain: A review. Spanish Journal of Agricultural Research. 15: 2171-9292.

11 Fredericksen F., Delgado F., Cabrera C., Yanez A., Gonzalo C. \& Villalba M. 2015. The effects of reference genes in qRT-PCR assays for determining the immune response of bovine cells (MDBK) infected with the Bovine Viral Diarrhea Virus 1 (BVDV-1). Gene. 569: 95-103.

12 Gomez-Romero N., Basurto-Alcantara F.J., Verdugo-Rodriguez A., Bauermann F.V. \& Ridpath J.F. 2017. Genetic diversity of bovine viral diarrhea virus in cattle from Mexico. Journal of Veterinary Diagnostic Investigation. 29: 362-365.

13 Gomez D.E., Arroyo L.G., Poljak Z., Viel L. \& Weese J.S. 2017. Detection of Bovine Coronavirus in Healthy and Diarrheic Dairy Calves. Journal of Veterinary Internal Medicine. 31: 1884-1891.

14 Ikuta N., De Oliveira Solla Sobral F., Lehmann F.K., da Silveira P.V., de Carli S. \& Casanova Y.S. 2014. Taqman real-time PCR assays for rapid detection of avian pathogenic Escherichia coli isolates. Avian Diseases. 58: 628-631.

15 Kailasan S., Halder S., Gurda B., Bladek H., Chipman P.R. \& McKenna R. 2015. Structure of an enteric pathogen bovine parvovirus. Journal of Virology. 89(3): 2603-2614.

16 Karikalan M., Rajukumar K., Mishra N., Kumar M., Kalaiyarasu S. \& Rajesh K. 2016. Distribution pattern of bovine viral diarrhoea virus type 1 genome in lymphoid tissues of experimentally infected sheep. Veterinary Research Communications. 40: 55-61.

17 Kuta A. \& Woźniakowski G.P. 2015. Cross-priming amplification for detection of bovine viral diarrhoea virus species 1 and 2. Journal of Applied Microbiology. 119: 632-639.

18 Lifang Y., Lanny W., Pace B.B., Floyd D.W. \& Zhang S. 2016. Failed detection of Bovine viral diarrhea virus 2 subgenotype a (BVDV-2a) by direct fluorescent antibody test on tissue samples due to reduced reactivity of field isolates to raw anti-BVDV antibody. Journal of Veterinary Diagnostic Investigation. 28(3): 150-157.

19 Liu H., Yang H., Zhao H., Li Y., Zhao H. \& Li T. 2015. Isolation and Identification of Bovine Parvovirus and its replication kinetics in Different Cells. Chinese Journal of Veterinary Science. 35: 1770-1776.

20 Mohamed F.F., Mansour S.M., El-Araby I.E., Mor S.K. \& Goyal S.M. 2017. Molecular detection of enteric viruses from diarrheic calves in Egypt. Archives of Virology. 162(2): 129-137.

21 Mohamed F.F., Mansour S.M.G., Orabi A., El-Araby I.E., Ng T.F.F. \& Mor S.K. 2018. Detection and genetic characterization of bovine kobuvirus from calves in Egypt. Archives of Virology. 163(7): 1439-1447.

22 Peddireddi L., Foster K.A., Poulsen E.G., An B., Hoang Q.H. \& O'Connell C. 2018. Molecular detection and characterization of transient bovine viral diarrhea virus (BVDV) infections in cattle commingled with ten BVDV persistently infected cattle. BMC Veterinary Research. 30: 413-22.

23 Qiu J., Soderlund-Venermo M. \& Young N.S. 2017. Human Parvoviruses. Clinical Microbiology Reviews. 30: 43-49.

24 Sakurai A., Nomura N., Nanba R., Sinkai T., Iwaki T. \& Obayashi T. 2011. Rapid typing of influenza viruses using super high-speed quantitative real-time PCR. Journal of Virological Methods. 178(1): 75-81.

25 Sareyy B. \& İbrahim B. 2017. Development of multiplex RT-PCR for detection and differentiationof foot-and-mouth disease virus $\mathrm{O}$ and A serotypes in Turkey. Turkish Journal of Veterinary And Animal Sciences. 41(5): 764-769.

26 Walraph J., Zoche-Golob V., Weber J. \& Freick M. 2018. Decline of antibody response in indirect ELISA tests during the periparturient period caused diagnostic gaps in Coxiella burnetii and BVDV serology in pluriparous cows within a Holstein dairy herd. Research in Veterinary Science. 118(2): 91-96.

27 Zhang S.Q., Tan B., Li P., Wang F.X., Guo L. \& Yang Y. 2014. Comparison of conventional RT-PCR reverse-transcription loop-mediated isothermal amplification and SYBR green I-based real-time RT-PCR in the rapid detection of bovine viral diarrhea virus nucleotide in con-taminated commercial bovine sera batches. Journal of Virological Methods. DOI: 10.1016/j.jviromet.2014.05.020. 
28 Zhang Y., Liu H., Wu X., Wang X., Li J. \& Zhao Y. 2015. A novel real-time RT-PCR with TaqMan-MGB probes and its application in detecting BVDV infections in dairy farms. Journal of Integrative Agriculture. 14(9): 1637-1643.

29 Zoccola R., Mazzei M., Carrozza M.L., Ricci E., Forzan M. \& Pizzurro F. 2017. A newly developed BVDV-1 RTqPCR Taqman assay based on Italian isolates: evaluation as a diagnostic tool. Folia Microbiologica. 62(2): 279-286. 